\title{
Liquid Crystal-based Imaging of Enzymatic Reactions at Aqueous-liquid Crystal Interfaces Decorated with Oligopeptide Amphiphiles
}

\author{
Qiongzheng Hu and Chang-Hyun Jang* \\ College of BioNano Technology, Kyungwon University, Seongnam-City, Gyeonggi-Do 461-701, Korea \\ *E-mail: chjang4u@kyungwon.ac.kr \\ Received February 1, 2010, Accepted March 9, 2010
}

\begin{abstract}
In this study, we investigated the use of liquid crystals to selectively detect the activity of enzymes at interfaces decorated with oligopeptide-based membranes. We prepared a mixed monolayer of tetra(ethylene glycol)-terminated lipids and carboxylic acid-terminated lipids at the aqueous-liquid crystal (LC) interface. The 17 amino-acid oligopeptide SNFKTIYDEANQFATYK was then immobilized onto this mixed monolayer through N-hydroxysuccinimide-activation of the carboxylic acid groups. We examined the orientational behavior of nematic 4-cyano-4'-pentylbiphenyl (5CB) after conjugation of the 17 amino-acid oligopeptide with the mixed monolayer assembled at the interface. Immobilization of the oligopeptide caused orientational transitions in 5CB, with a change from homeotropic (perpendicular) to tilted alignment, which was primarily due to the reorganization of the monolayer. The orientation of the 5CB molecules returned to its homeotropic state after contacting the interface containing $\alpha$-chymotrypsin, which can cleave the immobilized oligopeptide. Control experiments confirmed that the enzymatic activity of $\alpha$-chymotrypsin triggered the ordering transitions in the LC. These results suggest that the LC can provide a facile method for selective detection of enzymatic activity.
\end{abstract}

Key Words: Liquid crystals, 4-Cyano-4'-pentylbiphenyl (5CB), Orientational behavior, Oligopeptide-based membranes, Enzymatic activity

\section{Introduction}

Biomolecular interactions occurring at biological membranes represent a field of study that concerns the binding of proteins, ${ }^{1,2}$ enzymatic events, ${ }^{3}$ and viral infections, ${ }^{4}$ and their role in controlling biochemical processes. In recent years, various attempts have been made to provide convenient and simple methods of sensing these biomolecular interactions by exploiting the selfassembly properties of the constituents of biological membranes at interfaces. ${ }^{1-9}$ However, widespread use of these approaches has been limited because they generally require the use of labeled molecules, which largely complicate the surface-based assays and prevent high levels of multiplexing.

A promising method for avoiding this limitation is to use liquid crystalline materials. The orientational properties of liquid crystals (LCs) enables the amplification and the transduction of biologically relevant binding events at nanostructured surfaces into optical outputs. ${ }^{10-12}$ These signals can be used to image receptor-mediated binding of proteins at surfaces with submicrometer spatial resolution. ${ }^{13,14}$ This method is potentially useful because it does not require the use of enzymatic or fluorescent labels. ${ }^{15,16}$ In addition, this approach does not require the use of complex instrumentation ${ }^{17}$ or laborious techniques. ${ }^{18}$

Abbott et al. have shown that phospholipids assembled at aqueous-LC interfaces couple strongly to the orientation of the LC, and the binding of proteins at these interfaces induces ordering transitions in the LC. ${ }^{19-23}$ The activities of the proteins could be easily observed between crossed polarizers because the longrange orientational order in the LC couples to the organization of the proteins and phospholipids at the interface.

Recently, Park et al. reported on a new strategy that enables the decoration of aqueous-LC interfaces with oligopeptidebased amphiphiles. ${ }^{24,25}$ They showed that covalent attachment of the oligopeptide to the NHS-activated carboxylic acid groups of the lipid-laden monolayer led to an ordering transition in the $\mathrm{LC}$; in addition, they demonstrated that the orientation of the LC at this interface could be selectively manipulated by contacting the oligopeptide-decorated LC with an enzyme that can cleave the immobilized oligopeptides. ${ }^{24,25}$

This study was conducted to test the possibility of using this method as a general enzyme assay. The approach used in this study builds from the results of an experimental system that provides approximately planar and stable interfaces between aqueous phases and the LCs. The 17 amino-acid oligopeptide SNFKTIYDEANQFATYK (Figure 1A) used in this study possesses two lysine residues and a primary amino group at the $\mathrm{N}$-terminus, and thus could form up to 3 covalent bonds to $\mathrm{N}$ hydroxysuccinimide (NHS)-activated carboxylic acid groups presented at this interface (Figure 1B). Park et al. previously confirmed the successful conjugation of the oligopeptide to NHS-activated carboxylic acid groups presented at this interface using polarized modulation infrared reflection-adsorption spectroscopy (PM-IRRAS). ${ }^{25}$ We used $\alpha$-chymotrypsin as a model enzyme that can slice the oligopetide at four different locations. We found that $\alpha$-chymotrypsin selectively cleaved peptide bonds after phenylananine (F) and tyrosine (Y) on the C-terminal side in the 17 amino-acid oligopeptide.

\section{Experimental Details}

Materials. The nematic liquid crystal 4-cyano-4'-pentylbiphenyl (5CB), manufactured by BDH, was purchased from EM 
(A)

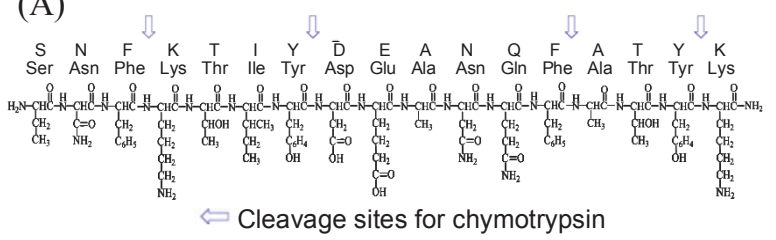

(B)

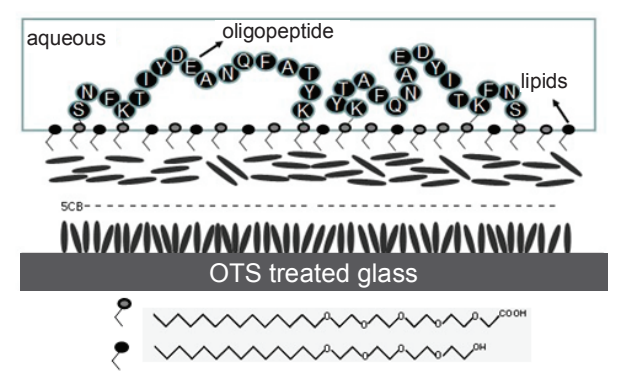

Figure 1. (A) The chemical structure of the 17-amino acid oligopeptide and (B) schematic illustration of the 17-amino-acid oligopeptide immobilized on the mixed monolayer of tetra(ethylene glycol)-terminated and carboxylic acid-terminated lipid at the aqueous-liquid crystal interface.

Industries (Hawthorne, NY). The glass microscope slides were Fisher's Finest, premium grade slides obtained from Fisher Scientific (Pittsburgh, PA). $20 \mu \mathrm{L}$ capillary and eight-well chamber slides were also obtained from Fisher Scientific. Copper specimen grids with 50 meshes, bar width of $80 \mu \mathrm{m}$, and hole width $420 \mu \mathrm{m}$ were purchased from GILDER. Octyltrichlorosilane (OTS), methanol, methylene chloride, sulfuric acid, hydrogen

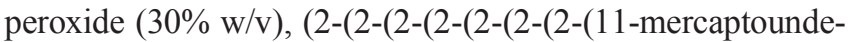
cyloxy)ethoxy)ethoxy)ethoxy)ethoxy)ethoxy)ethoxy) acetic acid, tetra (ethyl glycol) monotetradecyl ether, heptane, N-hydroxysuccinimide (NHS), 1-ethyl-2-(3-dimethylaminopropyl) carbodiimide hydrochloride (EDC), ethanolamine, $\alpha$-chymotrypsin, trypsin-chymotrypsin inhibitor, bovine serum albumin (BSA), phosphate buffered saline (PBS) $(10 \mathrm{mM}$ phosphate, $138 \mathrm{mM} \mathrm{NaCl}, 2.7 \mathrm{mM} \mathrm{KCl} ; \mathrm{pH}=7.4$ ), tris buffer saline (TBS) (50 mM Tris, $138 \mathrm{mM} \mathrm{NaCl}, 2.7 \mathrm{mM} \mathrm{KCl} ; \mathrm{pH}=8.0$ ), and calcium chloride dihydrate were all obtained from Sigma-Aldrich. The 17-amino-acids used in this study were purchased from PEPTRON (Seoul, Korea). All aqueous solutions were prepared with high purity deionized water $(18 \mathrm{M} \Omega \mathrm{cm})$ using a Milli-Q water purification system (Millipore, Bedford, MA).

Treatment of glass microscope slides with OTS. The glass microscope slides were cleaned using a "piranha solution" ( $70 \% \mathrm{H}_{2} \mathrm{SO}_{4} / 30 \% \mathrm{H}_{2} \mathrm{O}_{2}$, Caution: "piranha solution" reacts violently with organic materials and should be handled with extreme caution; do not store the solution in closed containers) for 1 hour at $80^{\circ} \mathrm{C}$. The slides were then rinsed with water, ethanol, and methanol, and dried under a stream of gaseous $\mathrm{N}_{2}$, followed by heating to $120^{\circ} \mathrm{C}$ overnight prior to OTS deposition. The "piranha-cleaned" glass slides were immersed into an OTS $/ n$-heptane solution for 30 minutes. The slides were then rinsed with methylene chloride and dried under a stream of $\mathrm{N}_{2}$. The OTS slides were tested for homeotropic alignment by observing the orientation of $5 \mathrm{CB}$ sandwiched between two OTS slides. Any slide not displaying homeotropic alignment was discarded.

Preparation of optical cells. The OTS-coated glass slides were fixed at the bottom of each well of an eight-well chamber slide. Copper specimen grids were then placed onto the OTScoated glass slide. $1.5 \mu \mathrm{L}$ of $5 \mathrm{CB}$ (heated at $50{ }^{\circ} \mathrm{C}$ for 15 minutes) was dispensed onto each grid and the optical cell was heated to $50^{\circ} \mathrm{C}$ (above the nematic-isotropic transition temperature of $5 \mathrm{CB}, \sim 35^{\circ} \mathrm{C}$ ). The excess $\mathrm{LC}$ was removed by contacting a $20 \mu \mathrm{L}$ capillary tube with the $5 \mathrm{CB}$ droplet on the grid. This procedure led to the formation of a stable film of $5 \mathrm{CB}$ within the grid. Subsequently, the optical cell was heated to $50{ }^{\circ} \mathrm{C}$ again and then immediately immersed in $500 \mu \mathrm{L}$ aqueous solution of interest held at room temperature $\left(\sim 20^{\circ} \mathrm{C}\right)$. The surface of the $5 \mathrm{CB}$ in contact with the aqueous phase was approximately flat as determined by concurrent focus of the grid and $5 \mathrm{CB}$ under an optical microscope.

Optical examination of LC textures. A polarized light microscope (ECLIPSE LV100POL, Nikon, Tokyo, Japan) was used to image the optical textures formed by polarized light transmitted through the optical cells filled with nematic $5 \mathrm{CB}$. All images were obtained using a $4 \times$ objective lens between crossed polarizers. Imaging the optical appearance of the $\mathrm{LC}$ was made with a digital camera (DS-2Mv, Nikon, Tokyo, Japan) that was attached to the polarized light microscope. The images were captured at a resolution of $1600 \times 1200$ pixels, a gain of $1.00 \times$, and a shutter speed of $1 / 10 \mathrm{~s}$.

Preparation of the mixed lipids and the 17-amino-acid oligopeptide solutions. To prepare a mixed monolayer of lipids at the aqueous/LC interface, a mixture of 3, 6, 9, 12, 15-pentaoxanonacosanoic acid and tetra (ethylene glycol) monotetradecyl ether was dissolved in a PBS buffer $(0.8 \mathrm{mM}$ and $0.2 \mathrm{mM}$, respectively). The mixed lipid solutions were confirmed to be stable for at least one week. The 17-amino-acid oligopeptide was prepared in a PBS solution stirred in a water-bath at $50^{\circ} \mathrm{C}$ for 4 hours. The oligopeptide solutions were vortexed for 1 minute immediately before use.

\section{Results and Discussion}

Formation of mixed monolayer of lipids at aqueous/LC interface. Past studies have demonstrated that this approach permits the formation of stable and approximately planar interfaces between water immiscible LC and an aqueous phase that can be readily exchanged. ${ }^{19-23}$ Before the aqueous solution was immersed into the optical cell, 5CB confined in the copper grid was directly contacted with air. The dark appearance of the LC between crossed microscopic polarizers was observed because OTStreated glass slides anchor the $5 \mathrm{CB}$ in a homeotropic orientation (perpendicular orientation to the surface) at the interface. After introduction of the PBS solution, the optical appearance of 5CB became bright and colorful (Figure 2A), which reflects in-plain birefringence that was associated with a planar or tilted orientation of the $\mathrm{LC}$ at the interface. We next adsorbed the mixture of lipids from an aqueous solution of $0.8 \mathrm{mM} 3,6,9,12,15$ pentaoxanonacosanoic acid $\mathrm{CH}_{3}\left(\mathrm{CH}_{2}\right)_{13}\left(\mathrm{OCH}_{2} \mathrm{CH}_{2}\right)_{4} \mathrm{OCH}_{2}$ $\mathrm{CO}_{2} \mathrm{H}$ and $0.2 \mathrm{mM}$ tetra(ethylene glycol) monotetradecyl ether $\mathrm{CH}_{3}\left(\mathrm{CH}_{2}\right)_{13}\left(\mathrm{OCH}_{2} \mathrm{CH}_{2}\right)_{4} \mathrm{OH}$ in the PBS by replacing the PBS 


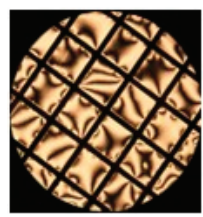

(A)

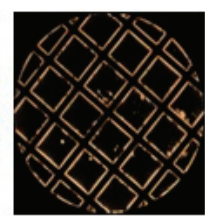

(B)

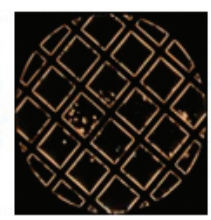

(C)

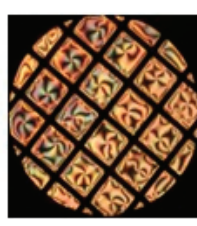

(D)
Figure 2. Optical images of $5 \mathrm{CB}$ viewed through a polarized light microscope: (A) incubation with the PBS buffer $(\mathrm{pH}=7.4)$, (B) after $1 \mathrm{~h}$ of incubation with an aqueous mixture of $0.8 \mathrm{mM} \mathrm{3}, 6,9,12,15$ pentaoxanonacosanoic acid $\mathrm{CH}_{3}\left(\mathrm{CH}_{2}\right)_{13}\left(\mathrm{OCH}_{2} \mathrm{CH}_{2}\right)_{4} \mathrm{OCH}_{2} \mathrm{CO}_{2} \mathrm{H}$ and $0.2 \mathrm{mM}$ tetra(ethylene glycol) monotetradecyl ether $\mathrm{CH}_{3}\left(\mathrm{CH}_{2}\right)_{13}$ $\left(\mathrm{OCH}_{2} \mathrm{CH}_{2}\right)_{4} \mathrm{OH}$ in PBS, (C) after $0.5 \mathrm{~h}$ of reaction with EDC/NHS, and (D) after $2 \mathrm{~h}$ of incubation with aqueous solution of the 17-aminoacid oligopeptide (SNFKTIYDEANQFATYK).

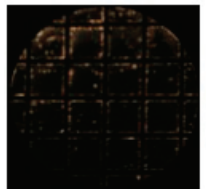

(A)

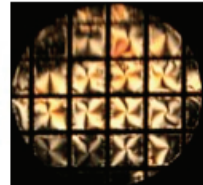

(B)

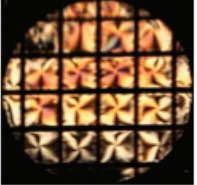

(C)

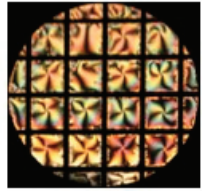

(D)
Figure 3. Optical images of $5 \mathrm{CB}$ viewed through a polarized light microscope: (A) right after introduction of $20 \mathrm{mM}$ ethanolamine (PBS buffer, $\mathrm{pH}=7.4$ ), (B) after $0.5 \mathrm{~h}$ of incubation with the ethanolamine, (C) after $4 \mathrm{~h}$ of incubation with the ethanolamine, and (D) after removal of the free ethanolamine by exchanging the aqueous solution with TBS $(\mathrm{pH}=8.0) 5$ times.

solution in the optical cell. The entirely dark image (Figure 2B) indicates that there was an anchoring transition of the $\mathrm{LC}$ alignment from a planar to homeotropic state through the transfer of the lipids onto the aqueous-LC interface. Past studies have shown that the interaction between the aliphatic chains of lipids and the LCs can cause the homeotropic orientation of $5 \mathrm{CB}$ at the aqueous-LC interface. ${ }^{19-23}$ Although optical images darkened immediately after the introduction of the lipid mixture, the aqueous solution was maintained for 1 hour to allow for equilibration. We should point out that an unstable monolayer of lipids would appear if the copper grid was not completely clean or if the interface of $5 \mathrm{CB}$ and aqueous phase was not flat. In order to prevent the displacement of the $\mathrm{LC}$ from the grid, the meniscus must be kept on the interface while exchanging the aqueous solutions throughout the experiment.

Immobilization of oligopeptides to the mixed monolayer of lipids. The coupling was carried out by replacing the free lipid solution with an aqueous EDC/NHS $(200 \mathrm{mM} / 50 \mathrm{mM})$ in a PBS $(\mathrm{pH}=7.4)$ to activate the carboxylic acid groups presented at the interface. Previous study performed by Park et al. confirmed coupling of the amino-acid oligopeptides to activated $\mathrm{N}$-succinimidyl esters at a model interface. ${ }^{25}$ They used polarization modulation infrared reflection-absorption spectroscopy (PMIRRAS) and NHS-activated surfaces prepared by self-assembly of carboxylic acid-terminated alkanethiols $\left(\mathrm{HS}\left(\mathrm{CH}_{2}\right)_{11}-\left(\mathrm{OCH}_{2}\right.\right.$ $\left.\left.\mathrm{CH}_{2}\right)_{6}-\mathrm{OCH}_{2} \mathrm{CO}_{2} \mathrm{H}, \mathrm{EG}_{6}-\mathrm{CO}_{2} \mathrm{H}\right)$ on a flat gold surface.

Since the activated NHS ester has a short half-life in PBS buffer $(\mathrm{pH}=7.4)$, a fresh mixture of NHS and EDC was used each time. During activation for 0.5 hours, the optical appearance of $5 \mathrm{CB}$ remained dark (Figure 2C). This suggests that the NHS activation does not perturb the homeotropic orientation of $5 \mathrm{CB}$ at the aqueous-LC interface. The free EDC/NHS was washed out by exchanging the aqueous solution with the PBS five times; then, the PBS was replaced with a $0.05 \mathrm{mM}$ aqueous solution of the 17-amino-acid oligopeptide (SNFKTIYDEANQFATYK) in PBS. We predicted that the binding of multiple amino groups on the oligopeptide to the activated carboxylic acids would cause physical strain on the lipid membrane, thus disturbing the orientational of $5 \mathrm{CB}$. Within 2 hours of incubation with the oligopeptide solution, the optical appearance of $5 \mathrm{CB}$ gradually turned bright and colorful, which indicates that there was an anchoring transition of the $\mathrm{LC}$ from homeotropic to a tilted or planar alignment (Figure 2D). Control experiments confirmed that the optical appearance of $5 \mathrm{CB}$ did not change when the oligopeptide solution was in contact with the carboxylic acid solution in the absence of NHS/EDC activation.

Quenching the remaining NHS-activated carboxylic acid groups. Following the conjugation of the oligopeptides, we replaced the oligopeptide solution with $20 \mathrm{mM}$ ethanolamine in PBS to quench the remaining NHS-activated carboxylic acid groups. Park et al. reported that ethanolamines can form covalent bonds with the NHS-activated carboxylic groups; furthermore, the binding of ethanolamine did not perturb the orientational ordering of the LC. However, in our study, the bright optical appearance immediately turned black with the introduction of ethanolamine to the optical cell (Figure 3A). The dark appearance of $5 \mathrm{CB}$ gradually returned to a bright and colorful state when incubated in the ethanolamine solution for 4 hours (Figure 3B, C).

We hypothesize that the mechanism for this phenomenon was as follows: with the introduction of ethanolamine into the optical cell, the ethanolamine molecules reacted with the remaining NHS-activated carboxylic acid all at once, which would result in the expansion of the lipid monolayer and a conformational change of the 17-amino-acid oligopeptide. This reorganization would temporarily induce the ordering transition of the LC from a planar to homeotropic state. However, through the effect of torsional force, the oligopeptide would be inclined to return to its optimal conformation at the interface. When the multiple effects induced by ethanolamine and the torsion force reached a balanced equilibrium, the optical appearance of $5 \mathrm{CB}$ would be completely returned to its bright, colorful state (Figure $3 \mathrm{D})$. This bright image indicates the ordering transition of $5 \mathrm{CB}$ from a homeotropic to planar state.

Incubation with $\boldsymbol{\alpha}$-chymotrypsin. This study was conducted to test the hypothesis that enzyme reactions could be imaged with the LCs by designing interfaces that possess oligopeptidedecorated lipid membranes. It was assumed that the planar or tilted orientation of the liquid crystal was due to multiple points of interaction between the oligopeptide and the lipid membrane. Therefore, the planar orientation of $5 \mathrm{CB}$ at the oligopeptidedecorated lipid monolayer would revert to the homeotropic state upon contact with an enzyme that cleaves the immobilized oligopeptide substrate. In order to demonstrate this idea, we introduced $\alpha$-chymotrypsin into the optical cell, which can clea- 
(A)

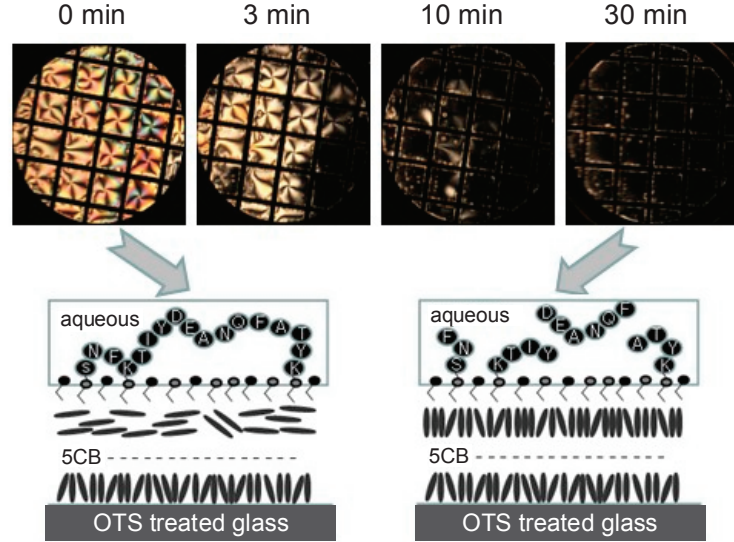

Figure 4. (A) Optical images of 5CB between crossed polarizers decorated with the oligopeptide after the introduction of $\alpha$-chymotrypsin, (B) schematic illustrations indicating the orientation of 5CB before (left) and after (right) the cleavage of the 17 amino-acid oligopeptide by $\alpha$-chymotrypsin.

(A)
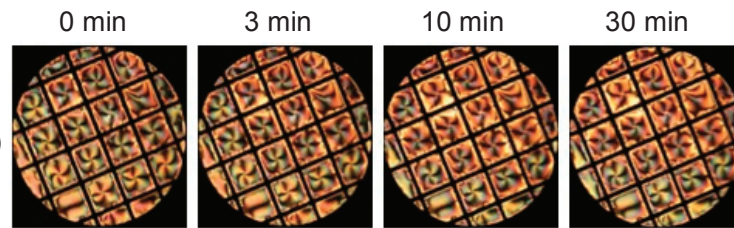

(B)
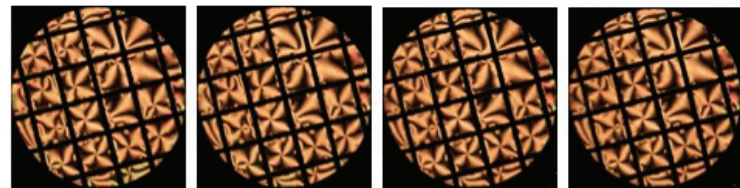

Figure 5. Optical images of $5 \mathrm{CB}$ viewed through a polarized light microscope: (A) after $30 \mathrm{~min}$ of incubation with the inhibitor-treated $\alpha$-chymotrypsin and (B) BSA solution.

ve the oligopeptide (SNFKTIYDEANQFATYK) at four different locations.

A $400 \mathrm{nM} \alpha$-chymotrypsin solution in $50 \mathrm{mM}$ TBS buffer $\left(\mathrm{pH}=8.0\right.$ ) containing $20 \mathrm{mM} \mathrm{Ca}^{2+}$ was prepared. The aqueous ethanolamine was washed out with TBS buffer five times and the $\alpha$-chymotrypsin solution was introduced into the optical cell. Within 3 minutes of contact with the $\alpha$-chymotrypsin solution, the interference color of $5 \mathrm{CB}$ changed and there was a gradual of the bright area, indicating a decrease in the tilted angle of $5 \mathrm{CB}$ at the aqueous-LC interface (Figure $4 \mathrm{~A}$ ). After 10 minutes of incubation, the optical appearance of $5 \mathrm{CB}$ became mostly black. This chemically specific reaction was completed within twenty minutes and a totally dark appearance was observed, which indicated that the orientation of $5 \mathrm{CB}$ had already reverted to a homeotropic state (Figure 4B).

We note that our method will also allow the quantitative analysis of enzyme activity by quantifying the tilt of $5 \mathrm{CB}$ at the aqueous-LC interface in the presence of the oligopeptide. The average tilt angles can be determined by measuring the retardance of the LCs using a microscope equipped with a retardance imaging system. The tilt angles of $5 \mathrm{CB}$ at the aqueous$\mathrm{LC}$ interface are calculated from the retardance and thickness of the LC. This study is under way in our laboratory. In our previous report we estimated the number density of virus particles on the solid-LC interface giving rise the orientational transition of LC to be lower than 1 virion/100 $\mathrm{m}^{2} .{ }^{14}$ Because LCs can be used to image regions of surfaces that are $\sim 100 \mu \mathrm{m}^{2}$, this results suggests that it may be possible to image single virion or small clusters of virions on the surfaces by using nematic LCs.

Control experiments. To test the hypothesis that the ordering transition of 5CB was induced by the enzymatic cleavage of the oligopeptide, we used a trypsin-chymotrypsin inhibitor (Bowman-Birk inhibitor). The inhibitor can block the enzymatic active sites of the enzyme by forming a 1:1 complex with chymotrypsin. The trypsin-chymotrypsin inhibitor was added to the $400 \mathrm{nM}$ solution of $\alpha$-chymotrypsin to give a 1:6 weight ratio of $\alpha$-chymotrypsin to the inhibitor. The mixed solution was incubated for 20 minutes at room temperature, and then introduced into the optical cell containing an oligopeptide-decorated interface on the liquid crystal. After incubating for more than 1 hour, no change in the orientational transition of $5 \mathrm{CB}$ was observed (Figure 5A). We also performed a control experiment with bovine serum albumin (BSA), which is incapable of cleaving the oligopeptide substrate. As expected, we observed no ordering transition in the LC after 1 hour of incubation with BSA (Figure 5B). Based on these results, we concluded that the orientational transition of the $\mathrm{LC}$ was induced by the enzymatic reaction of $\alpha$-chymotrypsin, which specifically cleaved the 17 amino-acid oligopeptide substrate, rather than by the non-specific interactions.

\section{Conclusion}

This study demonstrates that LCs can be used to selectively detect the activity of enzymes at interfaces decorated with oligopeptide-based membranes. The orientation of the LC at the peptide-decorated interface could be selectively manipulated by the introduction of an enzyme that can cleave the immobilized oligopeptide substrates. Control experiments revealed that the orientational transition of the LC was induced by the enzymatic reaction of $\alpha$-chymotrypsin, which specifically cleaved the 17 amino-acid oligopeptide substrate, rather than non-specific interactions. Therefore, the results of this study establish the generality of using peptide-decorated interfaces as substrates for selective detection of enzymatic activity that can be imaged by the LC.

Acknowledgments. This work was supported by the Kyungwon University Research Fund in 2010, the grant from KRIBB Research Initiative Program, and the grant from Korea Small and Medium Business Administration in 2010 (Grant No. 00037812).

\section{References and Notes}

1. Song, X.; Swanson, B. I. Anal. Chem. 1999, 71, 2097.

2. Dietrich, C. Biophys. J. 2001, 80, 1417.

3. Stahelin, R. V.; Cho, W. Biochem. J. 2001, 359, 679.

4. Charych, D. H.; Nagy, J. O.; Spevak, W.; Bednarski, M. D. Science 1993, 261, 585.

5. Tanaka, K.; Manning, P. A.; Yu, H. Langmuir 2000, 16, 2665. 
6. Yang, T.; Jung, S. Y.; Mao, H.; Cremer, P. S. Anal. Chem. 2001, $73,165$.

7. Gast, A. P.; Robertson, C. R.; Wang, S. W.; Yatcilla, M. T. Biomol. Eng. 1999, 16, 21.

8. Park, K. S.; Shin, S. Y.; Hahm, K. S.; Kim, Y. M. Bull. Korean Chem. Soc. 2003, 24, 1478.

9. Kim, C. Bull. Korean Chem. Soc. 2010, 31, 372.

10. Gupta, V. K.; Skaife, J. J.; Dubrovsky, T. B.; Abbott, N. L. Science 1998, 279, 2077.

11. Tingey, M. L.; Wilyana, S.; Snodgrass, E. J.; Abbott, N. L. Langmuir 2004, 20, 6818.

12. Jang, C. H.; Tingey, M. L.; Korpi, N. L.; Abbott, N. L. J. Am. Chem. Soc. 2005, 127, 8912.

13. Skaife, J. J.; Abbott, N. L. Langmuir 2000, 16, 3529.

14. Jang, C. H.; Cheng, L. L.; Olsen, C. W.; Abbott, N. L. Nano Lett. 2006, 6(5), 1053.

15. Nielsen, U. B.; Cardone, M. H.; Sinskey, A. J.; MacBeath, G.; Sorger, P. K. Proc. Natl. Acad. Sci. U.S.A. 2003, 100, 9330.
16. Harlow, E.; Lane, D. Antibodies: A Laboratory Manual. Cold Spring Harbor Laboratory; New York, 1998.

17. Pandey, A.; Mann, M. Nature 2000, 405, 837.

18. Vénien, A.; Levieux, D.; Dufour. E. Colloid Interface Sci. 2000, 223, 215.

19. Brake, J. M.; Daschner, M. K.; Luk, Y.Y.; Abbott, N. L. Science (Washington, D.C.) 2003, 302, 2094.

20. Lockwood, N. A.; Abbott, N. L. Curr. Opin. Colloid Interface Sci. 2005, 10, 111.

21. Brake, J. M.; Mezera, A. D.; Abbott, N. L. Langmuir 2003, 19, 8629.

22. Brake, J. M.; Daschner, M. K.; Abbott, N. L. Langmuir 2005, 21, 2218.

23. Lockwood, N. A.; de Pablo, J. J.; Abbott, N. L. Langmuir 2005, 21,6805 .

24. Park, J. S.; Teren, S.; Tepp, W. H.; Beebe, D. J.; Johnson, E. A.; Abbott, N. L. Chem. Mater. 2006, 18, 6147.

25. Park, J. S.; Abbott, N. L. Adv. Mater. 2008, 20, 1185. 